
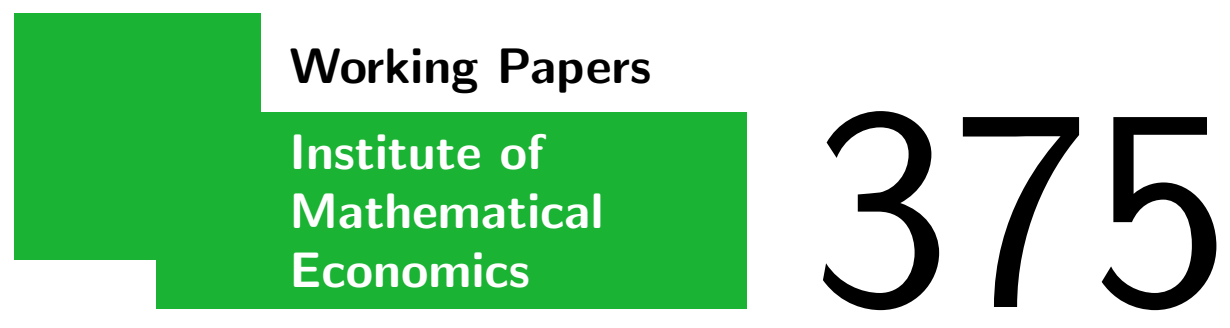

November 2005

\title{
Government versus Opposition: Who Should be Who in the 16th German Bundestag?
}

Dinko Dimitrov and Claus-Jochen Haake

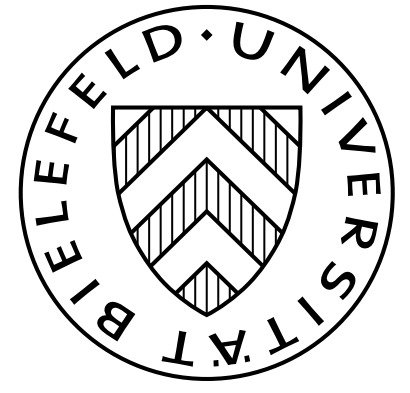

IMW · Bielefeld University

Postfach 100131

33501 Bielefeld · Germany

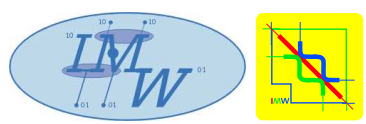

email: imw@wiwi.uni-bielefeld.de

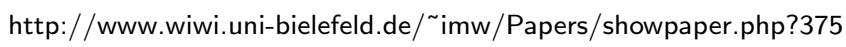

ISSN: 0931-6558 


\title{
Government versus Opposition: Who Should be Who in the 16th German Bundestag?*
}

\author{
Dinko Dimitrov and Claus-Jochen Haake \\ Institute of Mathematical Economics, Bielefeld University \\ P.O. Box 100131, 33501 Bielefeld, Germany \\ Emails: d.dimitrov@wiwi.uni-bielefeld.de, chaake@wiwi.uni-bielefeld.de
}

November 21, 2005

\begin{abstract}
We model the process of coalition formation in the 16th German Bundestag as a hedonic coalition formation game. In order to induce players' preferences in the game we apply the Shapley value of the simple game describing all winning coalitions in the Bundestag. Using different stability notions for hedonic games we prove that the "most" stable government is formed by the Union Parties together with the Social Democratic Party.

JEL Classification: D72, C71.
\end{abstract}

Keywords: coalition formation, Shapley value, simple games, winning coalitions.

\section{Introduction}

In this paper we address the question which parties in the 16th German Bundestag "should" form a government. According to the election results from September 18, 2005, there are five fractions obtaining seats in the parliament. From the distribution of seats, one can easily identify those coalitions of parties that are winning in the sense that they have a majority and, therefore, may agree to form the government. However, as past negotiations within different constellations have shown, each party clearly has preferences over the possible governments

${ }^{*}$ Financial support from the Alexander von Humboldt Foundation (D. Dimitrov) is gratefully acknowledged. 
it may be a member of. On the one hand this has of course to do with political views, but on the other hand, each party also looks at its "strength" in a government. Strength can, for example, be quantified by the number of ministries that this party would obtain. In view of this, any government can be tested on its stability in the sense that there is no other government that is preferred to the tested one by any of its members.

What would be an appropriate analytical tool to answer the question who should be government and who should be opposition? Using the language of cooperative game theory, and taking the distribution of seats in the parliament into account, we have a well defined simple game (cf. Shapley (1962)) with the parliamentary fractions in the Bundestag as players. The game itself only reflects the possibilities to form majorities. What we need to know is how ministries will be distributed among parties in a government. Hence, we need a tool to measure the "power" of a party. Here we use the Shapley value (Shapley (1953)) $)^{1}$ as a solution concept on simple games that reflects parties strengths, taking their opportunities into account to be part of majorities. By using the Shapley value, we assume parties to be purely office seeking and fade out political affinities.

Assuming that each party's incentive to take part in a government depends on how much power it is assigned by the Shapley value, we obtain preferences over coalitions. These preferences together form a hedonic coalition formation game (cf. Banerjee et. al. (2001) and Bogomolnaia and Jackson (2002)). A solution of this (and each) hedonic game is a partition of the set of parties into coalitions, which in particular assigns to each party its role in the government or in the opposition. Moreover, we have a suitable environment in which the question of stability can be approached. As it can be easily seen, it is not possible a coalition structure to be stable (to be defined later) if it does not contain a winning coalition. Hence, the answer to the question which partitions are stable is at the same time an answer to the question which government should form with respect to stability concerns.

The paper is organized as follows. In Section 2 we introduce some basic notions from the theory of simple games and hedonic games, and discuss different stability concepts for hedonic games (see also Sung and Dimitrov (2005)). In Section 3 we first define the simple game that corresponds to the distribution of seats in the 16th German Bundestag and then induce a hedonic game by using the Shapley value of all subgames of the simple game. As it turns out, the strongest form of stability that can be achieved in this case is semistrict core stability. Moreover, all partitions in the semistrict core share a common property: in each of them the government is formed by the Union Parties together with the Social Democratic Party. Thus, the analysis along the above ideas concurs with the actual result from the current government finding process. We conclude in Section 4 by relating our results to the study of coalition formation in simple games provided by Shenoy (1979) and Kirchsteiger and Puppe (1997).

\footnotetext{
${ }^{1}$ In the context of simple games also known as the Shapley-Shubik Index.
} 


\section{Preliminaries}

\subsection{Simple Games}

A simple game with transferable utility (or simple TU-game) is a pair $(N, v)$, where $N=$ $\{1, \ldots, n\}$ denotes the set of players and $v: 2^{N} \rightarrow\{0,1\}$ satisfying $v(\emptyset)=0$ is called a characteristic function. Subsets $S \subseteq N$ will be called coalitions. In the context of parliamentary fractions forming coalitions, we may distinguish coalitions that are "strong enough" to form a majority and those who are not. Consequently, we interpret a value $v(S)=1$ as coalition $S$ having a majority in the parliament (or being able to win an election). Analogously, $v\left(S^{\prime}\right)=0$ means that $S^{\prime}$ cannot win in a (majority) decision. For a simple game $(N, v)$, we denote by $\mathcal{W}^{(N, v)}=\{S \subseteq N \mid v(S)=1\}$ the set of winning coalitions and by $\mathcal{M} \mathcal{W}^{(N, v)}=\{S \subseteq N \mid v(S)=1$ and $v(T)=0$ for all $T \subset S\}$ the set of minimal winning coalitions, i.e., those winning coalitions that cannot be further reduced without losing the majority.

A simple game $(N, v)$ is monotonic if $v(S)=1$ implies $v(T)=1$ for all $T \supseteq S$, and proper if $v(S)=1$ implies $v(N \backslash S)=0$. For each $S \subseteq N$ the subgame $\left(S, v_{\mid S}\right)$ is obtained from $(N, v)$ by restricting attention to $S$, i.e., $v_{\mid S}(T)=v(T)$ for all $T \subseteq S$. The set of all simple TU-games on the player set $N$ will be denoted by $\mathcal{G}^{N}$.

Finally, a solution (of a simple game) is a mapping $f: \mathcal{G}^{N} \rightarrow \mathbb{R}^{N}$ that satisfies $\sum_{i \in N} f_{i}(v) \leq$ $v(N)$. In the context of simple games, $f$ shall readily be interpreted as an "index of power" as, in case $v$ is not the zero game, a total value of 1 is distributed among the players. The most prominent solution concept is undoubtedly the Shapley value (Shapley (1953)). The Shapley value of player $i$ in a game $(N, v)$ is given by the formula

$$
S h_{i}(N, v)=\sum_{S \subseteq N} \frac{(|N|-|S|) !(|S|-1) !}{|N| !}(v(S)-v(S \backslash\{i\})) \quad(i \in N) .
$$

\subsection{Hedonic Games}

Again, consider a finite set of players $N=\{1, \ldots, n\}$ and for each player $i \in N$, we denote by $\mathcal{N}_{i}=\{X \subseteq N \mid i \in X\}$ the collection of all coalitions containing $i$. A partition $\Pi$ of $N$ into coalitions is called a coalition structure, i.e., the coalitions in $\Pi$ are pairwise disjoint and $\bigcup_{X \in \Pi} X=N$. For each coalition structure $\Pi$ and each player $i \in N$, by $\Pi(i)$ we denote the coalition in $\Pi$ containing $i$, i.e., $\Pi(i) \in \Pi$ and $i \in \Pi(i)$.

We assume that each player $i \in N$ is endowed with a preference $\succeq_{i}$ over $\mathcal{N}_{i}$, i.e., a binary relation over $\mathcal{N}_{i}$ which is complete and transitive. By $\succ_{i}$ and $\sim_{i}$ we denote the strict and the equivalence relation corresponding to $\succeq_{i}$. We write $\succeq=\left(\succeq_{1}, \ldots, \succeq_{n}\right)$ for the profile of 
preferences $\succeq_{i}(i \in N)$. Player $i$ 's preference over coalitions naturally induces a preference over coalition structures in the following way: Coalition structure $\Pi$ is weakly preferred to $\Pi^{\prime}$ if and only if $\Pi(i) \succeq \Pi^{\prime}(i)$ holds. In this sense $i$ 's preference over coalition structures is purely hedonic, as he only cares about the coalition he is a member of.

A hedonic game $(N, \succeq)$ is a pair of a finite set $N$ of players and a preference profile $\succeq$ as above. In contrast to TU games, a hedonic game does not provide incentives for players to form coalitions by assigning a worth to each coalition, but it reflects player's preferences to join certain coalitions. Hence, a solution for a hedonic game shall not distribute some value among the players but should analyze which coalitions should form on the basis of players' preferences. In effect it asks for which coalition structure evolves. To this end, stability notions (with respect to coalition structures) enter the scene. We next discuss three different versions.

Let $(N, \succeq)$ be a hedonic game. For any coalition $X \subseteq N$ and for any coalition structure $\Pi$ of $N$, let $\mathcal{X}^{\Pi}(X):=\{X \cap P \mid P \in \Pi\}$. We say that $\Pi$ is strictly core stable if there does not exist a nonempty coalition $X$ such that $X \succeq_{i} \Pi(i)$ holds for all $i \in X$ and $X \succ_{j} \Pi(j)$ is true for some player $j \in X . \Pi$ is semistrictly core stable if there does not exist a nonempty coalition $X$ such that $X \succeq_{i} \Pi(i)$ for all $i \in X$ and for each $X^{\prime} \in \mathcal{X}^{\Pi}(X)$ there is $j \in X^{\prime}$ with $X \succ_{j} \Pi(j)$. $\Pi$ is core stable if there does not exist a nonempty coalition $X$ such that $X \succ_{i} \Pi(i)$ holds for each $i \in X$.

Put in other words, a coalition structure $\Pi$ is strictly core stable if there is no coalitional deviation in which every player is weakly better off and at least one player is strictly better off in comparison to the corresponding coalitions in $\Pi$. In the definition of semistrict core stability a more precise structure of the set of players who are strictly better off in a deviation is added; this addition is made by requiring that at least one player from each original coalition (according to $\Pi$ ) strictly prefers to be in the corresponding deviating coalition.2 Clearly, the weakest notion of a coalitional deviation is incorporated in the definition of core stability - everyone in the deviating coalition should be strictly better off.

To conclude, we mention the simple observation that strict core stability implies semistrict core stability that, in turn, implies core stability.

\section{Coalition Formation via the Shapley Value}

In this section we address the question which parties in the 16th German Bundestag should form the government and who should form the opposition, if arguments for or against are based on stability concepts as introduced in the previous section. Observe that the distribu-

\footnotetext{
${ }^{2}$ The idea of semistrict core stability can already be found in the work of Kirchsteiger and Puppe (1997).
} 
tion of seats in the parliament naturally defines a simple game that reflects all opportunities to form majorities. In the current parliament there are five fractions present (listed according to the number of seats): (1) CDU/CSU (Union Parties) with $s_{1}=226$ seats, (2) SPD (Social Democratic Party) with $s_{2}=222$ seats, (3) FDP (Liberal Democratic Party) with $s_{3}=61$ seats, (4) PDS.Die Linke (Left Party) with $s_{4}=54$ seats, and (5) B'90/Die Grünen (Green Party) with $s_{5}=51$ seats. To form a majority, a coalition thus requires a total of $614 / 2+1=308$ seats. Consequently, we may define a simple game $\left(N, v^{\text {Bundestag }}\right)=(N, v)$ with $N=\{1, \ldots, 5\}$ and $v$ be such that for all $S \subseteq N$ we have

$$
v(S)=1 \quad \text { if and only if } \quad \sum_{i \in S} s_{i} \geq 308 .
$$

The set of minimal winning coalitions is given by ${ }^{3}$

$$
\mathcal{M} \mathcal{W}^{(N, v)}=\{12,134,135,145,234,235,245\} .
$$

Notice also that $(N, v)$ is monotonic and proper.

$(N, v)$ alone only reflects which coalitions may form a government. Yet, it does not include any information about how, e.g., ministries are to be distributed. The Shapley value measures the "strength" of a party within a coalition in the following way. Suppose the grand coalition (i.e., $N)$ agrees to form a government. The Shapley value of $(N, v)$, which is given by

$$
\operatorname{Sh}(N, v)=\frac{1}{30}(9,9,4,4,4),
$$

suggests, for example, that a "big" party (1 or 2 ) should get roughly twice as many ministries as a small one $(3,4$, or 5$)$.

Given the Bundestag game $(N, v)$, we define a hedonic game $\left(N, \succeq^{\text {Bundestag }}\right)=(N, \succeq)$ by inducing players' preferences over coalitions in the following way. For each $i \in N$ and for all $S, T \in \mathcal{N}_{i}$, we define $\succeq_{i}$ by

$$
S \succeq_{i} T \Leftrightarrow S h_{i}\left(S, v_{\mid S}\right) \geq S h_{i}\left(T, v_{\mid T}\right)
$$

According to (1), player $i$ 's preferences over any two coalitions $S$ and $T$ he is a member of are induced by $i$ 's Shapley value in the simple game restricted to $S$ and $T$, respectively. Notice that paying attention to the corresponding coalitions is compatible with the very definition of a hedonic game - each player in such a game evaluates any two coalition structures based only on his preferences over the coalitions in the two partitions he belongs to (cf. Aumann and Dréze (1974) and Shenoy (1979)).

We present below players' payoffs according to the Shapley value of the corresponding subgames (the first column) and players' preferences induced in the way indicated in (1).

\footnotetext{
${ }^{3}$ We simplify notation for coalitions by using, e.g., " 134 " instead of $\{1,3,4\}$.
} 
For example, player 1's payoff in the coalitions $\{1,2\}$ and $\{1,2,5\}$ is $S h_{1}\left(\{1,2\}, v_{\mid\{1,2\}}\right)=$ $S h_{1}\left(\{1,2,5\}, v_{\mid\{1,2,5\}}\right)=\frac{1}{2}$, and his payoff in the coalition $\{1,3,5\}$ is $S h_{1}\left(\{1,3,5\}, v_{\mid\{1,3,5\}}\right)=$ $\frac{1}{3}$. Hence, as displayed in Table 1 , each player is indifferent between any two coalitions on the same row, and each player strictly prefers a coalition on a higher row over a coalition on a lower row.

\begin{tabular}{c|l||l}
$S h_{i}$ & $\succeq_{1}$ & $\succeq_{2}$ \\
\hline \hline$\frac{1}{2}$ & $12,123,124,125,1345$ & $12,123,124,125,2345$ \\
$\frac{1}{3}$ & $134,135,145,1234,1235,1245$ & $234,235,245,1234,1235,1245$ \\
$\frac{9}{30}$ & 12345 & 12345 \\
0 & $1, \ldots$ & $2, \ldots$ \\
\hline
\end{tabular}

\begin{tabular}{c|l||l||l}
$S h_{i}$ & $\succeq_{3}$ & $\succeq_{4}$ & $\succeq_{5}$ \\
\hline \hline$\frac{1}{3}$ & $134,135,234,235$ & $134,145,234,245$ & $135,145,235,245$ \\
$\frac{1}{6}$ & $1234,1235,1345,2345$ & $1234,1245,1345,2345$ & $1235,1245,1345,2345$ \\
$\frac{4}{30}$ & 12345 & 12345 & 12345 \\
0 & $3, \ldots$ & $4, \ldots$ & $5, \ldots$ \\
\hline
\end{tabular}

Table 1: Preferences over coalitions

We now turn to the question whether there exist stable coalition structures for the 16th German Bundestag. As Proposition 11 shows, the strongest form of stability is not achievable.

Proposition 1 Let $(N, \succeq)$ be the hedonic game defined in (1). Then there is no strictly core stable coalition structure for $(N, \succeq)$.

It will be of benefit to present the proof of Proposition 1 after the proof of Proposition 2 .

Before we proceed, notice first that due to properness of $(N, v)$, any coalition structure can at most carry one winning coalition, which we refer to as government $(G)$. Analogously, the set of remaining players is called opposition $(O)$. The next lemma shows that splitting the opposition does not alter semistrict core stability.

Lemma 1 Let $\Pi=\{G, O\}$ be a coalition structure with two elements and for which $v(G)=1$ holds. If $\Pi$ is semistrictly core stable, then so is any coalition structure of the form $\Pi^{\prime}=$ $\left\{G, O_{1}, \ldots, O_{k}\right\}$, where $\left\{O_{1}, \ldots, O_{k}\right\}$ is a partition of $O$.

Proof. Suppose $\Pi^{\prime}=\left\{G, O_{1}, \ldots, O_{k}\right\}$ is not semistrictly core stable. Then there is a coalition $X \subseteq N$ such that for all $i \in X$ we have $X \succeq_{i} \Pi^{\prime}(i)$ and for each $X^{\prime} \in \mathcal{X}^{\Pi^{\prime}}(X)$ there is $j^{\prime} \in X^{\prime}$ with $X \succ_{j^{\prime}} \Pi\left(j^{\prime}\right)$. As $X$ has to be a winning coalition, we know by properness that $X \cap G \neq \emptyset$ and so there exist $i_{G} \in G \cap X, i_{O} \in \bigcup_{r=1}^{k} O_{r} \cap X=O \cap X$ with 
$X \succ_{i_{G}} \Pi^{\prime}\left(i_{G}\right)=\Pi\left(i_{G}\right)=G$ and $X \succ_{i_{O}} \Pi^{\prime}\left(i_{O}\right) \sim_{i_{O}} \Pi\left(i_{O}\right)=O$. Hence, $\Pi$ is not semistrictly core stable, which contradicts our assumption.

After obtaining a negative result for the strict core, we check, whether there are semistrictly core stable coalition structures.

Proposition 2 For the game $(N, \succeq)$ defined in (1) the coalition structure $\Pi=\{12,345\}$ is semistrictly core stable. Moreover, there are no semistrictly core stable coalition structures $\Pi^{\prime}=\left\{G, O_{1}, \ldots, O_{k}\right\}$ with $v(G)=1$ and $G \neq\{1,2\}$.

Proof. To see that the coalition structure $\{12,345\}$ is semistrictly core stable, notice that the coalition $\{1,2\}$ is among player 1's and player 2's best coalitions. Hence, for any $X \subseteq N$ with $X \cap\{1,2\} \neq \emptyset$ we have $\Pi(i) \succeq_{i} X$ for all $i \in X \cap\{1,2\}$. Recalling that any coalition $X \subseteq\{3,4,5\}$ cannot serve as a deviating coalition in the semistrict sense (since $v(X)=0$ holds) shows the semistrict core stability of $\{12,345\}$.

Let us now show that there are no other semistrictly core stable partitions of $N$. Clearly, any coalition structure that does not contain a winning coalition cannot be semistrictly core stable. Let $\Pi$ be a coalition structure and $G \in \Pi$ be the winning coalition in $\Pi$. Inspecting Table 1, it is immediately seen that $G$ can only be among the best coalitions for either player 1 or player 2 , because otherwise the coalition $\{1,2\}$ could form and both, 1 and 2 , would strictly benefit from that. It remains to distinguish two cases:

(a) If $G=\{1,2, k\} \in \Pi$ for some $k \in\{3,4,5\}$, then for the players in coalition $N \backslash\{2\}$ we have $N \backslash\{2\} \sim_{1}\{1,2, k\}$ and $N \backslash\{2\} \succ_{i} \Pi(i)$ for each $i \in N \backslash\{1,2\}$. Hence, $N \backslash\{2\}$ blocks $\Pi$.

(b) If $G=\{1,3,4,5\} \in \Pi$, then coalition $\{2,3,4\}$ may form, from which all of its members strictly benefit. Analogously, if $G=\{2,3,4,5\} \in \Pi$, then $\{1,3,4\}$ is a blocking coalition.

This completes the proof of Proposition 2 .

Proof of Proposition 1. In order to show that the strict core is empty, it is sufficient to check, whether there are semistrictly core stable coalition structures that are also strictly core stable. In view of Proposition 2, we have to show that there is no partition $\left\{O_{1}, \ldots, O_{k}\right\}$ of $\{3,4,5\}$, for which the partition $\left\{12, O_{1}, \ldots, O_{k}\right\}$ is strictly core stable.

Let $\Pi$ be an arbitrary coalition structure of this kind. Then consider, for example, coalition $X=\{1,3,4,5\}$ and notice that $X \sim_{1} \Pi(1)$ and $X \succ_{i} \Pi(i)$ for each $i \in\{3,4,5\}$, from which follows that $\Pi$ is not strictly core stable.

We can conclude from Propositions 1 and 2 that, if one pays attention to coalitional deviations, then the "most" stable coalition structures are those containing the coalition of 
Union Parties and Social Democrats as the government. Any other government coalition, e.g., the so-called "Jamaica coalition" containing CDU/CSU, FDP and the Green Party, are not (semistrictly core) stable, as both fractions in the "big" coalition (CDU/CSU and SPD) strictly benefit from objecting "Jamaica". With the same argument, one identifies the so-called "Ampel coalition" ("traffic light" coalition with SPD, FDP and the Green Party) not to be semistrictly core stable, either. In the end, from our analysis, there is good reason that exactly the government that is currently about to form in Germany should form.

Using the weakest stability concept that we introduced here, it turns out that the set of all core stable coalition structures with two elements is given by

$$
\{\{12,345\},\{123,45\},\{124,35\},\{125,34\}\} \text {. }
$$

With an appropriate version of Lemma 1, any core stable partition is either in the above set, or is obtained from an element above, in which the opposition is split.

Thus, the notion of core stability cannot distinguish between a government containing only the two big parties from one in which one of the small parties is also there. Clearly, in such a government, the small party would not obtain any ministry according to the Shapley value, because it is not needed. To this end, core stability is not strong enough to clearly answer the government formation question.

\section{Final Remarks}

The methodology used in this paper to assess government formation can of course be applied to a broader class of problems. Therefore, we close by relating our results to the study of coalition formation in simple games starting with the work of Shenoy (1979). Theorem 7.4 there provides a sufficient condition for nonemptiness of the core of an abstract game appropriately induced by a proper monotonic simple game. In order to explain this condition in our setting, let us take the winning coalitions $\{1,3,5\}$ and $\{1,3,4,5\}$, and mention that $S h_{1}\left(\{1,3,4,5\}, v_{\mid\{1,3,4,5\}}\right)=\frac{1}{2}>\frac{1}{3}=S h_{1}\left(\{1,3,5\}, v_{\mid\{1,3,5\}}\right)$. Thus, the Shapley value does not respect (in the simple game $(N, v)$ ) the fact that if players form a smaller winning coalition, then their power should not decrease since there are fewer players to share the same amount of power. In the words of Shenoy (1979), the simple game $(N, v)$ exhibits the paradox of smaller coalitions (with respect to the Shapley value), and exactly the absence of this paradox was shown by him to be a sufficient condition for nonemptiness of the core. In contrast, the simple game corresponding to the structure of the 16th German Bundestag exhibits this paradox and, nevertheless, even its semistrict core is nonempty. Moreover, as proved in Section 3, all partitions in the semistrict core share an interesting common property. 
On the other hand, one can see the players in the derived hedonic game as being motivated only by office-seeking considerations. A possible reason for such an interpretation is the fact that players' preferences over coalitions are based on their relative power as measured by the Shapley value. Given this interpretation, a comparison of our results with the corresponding results of Kirchsteiger and Puppe (1997) is needed. In particular, these authors study the process of coalition formation when parties are purely office-seeking, construct in an appropriate way a cooperative game with non-transferable utility, and prove that its strict core is nonempty and single valued provided that parties care only for their relative weight in a coalition (the weight being the number of votes for the corresponding party). In contrast, the semistrict core of the hedonic game we consider is nonempty, while the strict core is empty. The reason here is that in this paper we focus on the coalition formation process as being guided by the Shapley value of the corresponding simple games.

\section{References}

[1] Aumann, R. and J. Dréze (1974): Cooperative games with coalition structures, International Journal of Game Theory 3, 217-237.

[2] Bogomolnaia, A. and M. Jackson (2002): The stability of hedonic coalition structures, Games and Economic Behavior 38, 201-230.

[3] Banerjee, S., H. Konishi, and T. Sönmez (2001): Core in a simple coalition formation game, Social Choice and Welfare 18, 135-153.

[4] Kirchsteiger, G. and C. Puppe (1997): On the formation of political coalitions, Journal of Institutional and Theoretical Economics 153, 293-319.

[5] Shapley, L.S. (1962): Simple games: an outline of the descriptive theory, Behavioral Science 7, 59-66.

[6] Shapley, L.S. (1953): A value for $n$-person games, Annals of Mathematics Studies 28, $307-317$.

[7] Shenoy, P.P. (1979): On coalition formation: a game-theoretical approach, International Journal of Game Theory 8, 133-164.

[8] Sung, S.-C. and D. Dimitrov (2005): A taxonomy of myopic stability concepts for hedonic games, IMW Working Paper 373, Bielefeld University. 